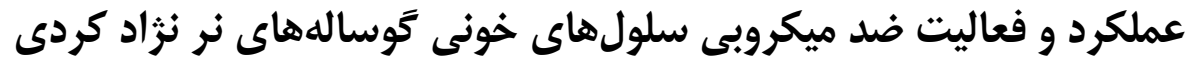

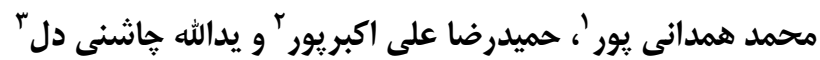

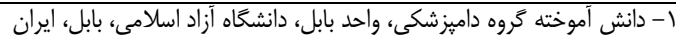

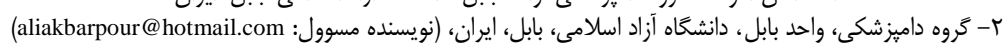

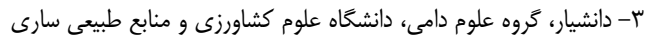

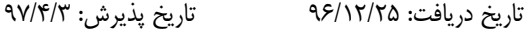

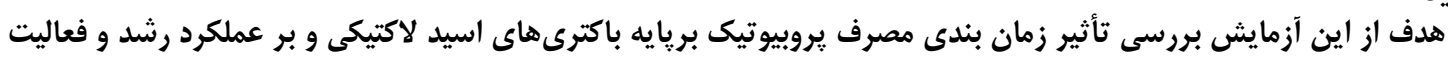

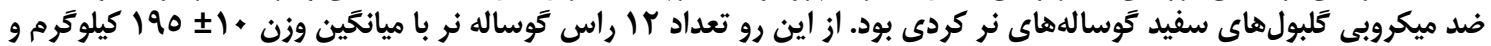

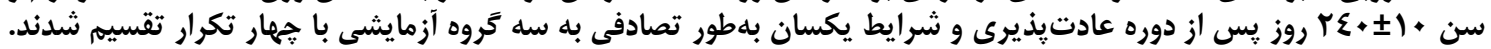

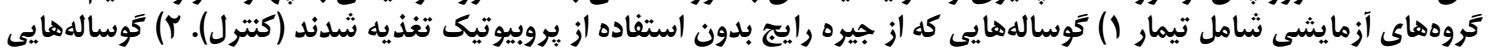

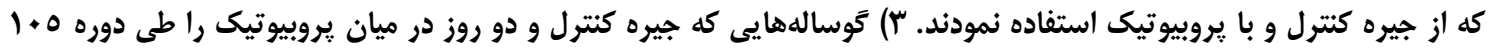

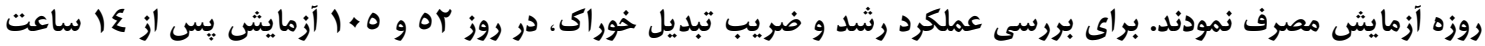

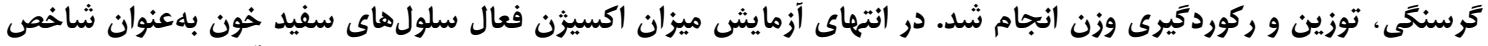

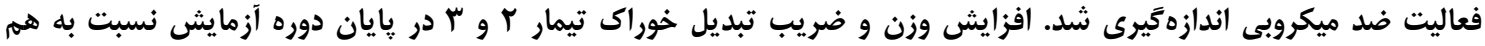

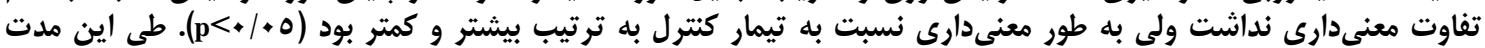

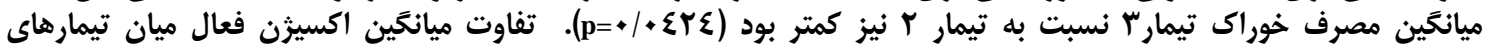

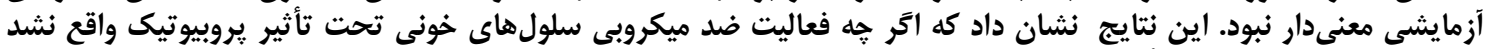

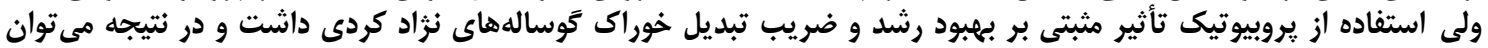

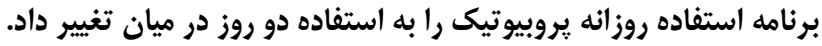

وازههاى كليدى: يروبيوتيك، اكسيزن فعال، رشد، توساله

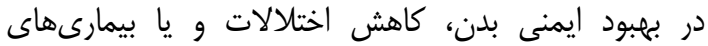

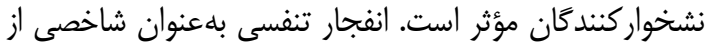

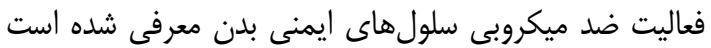

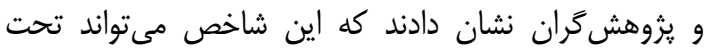

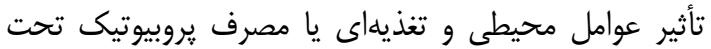

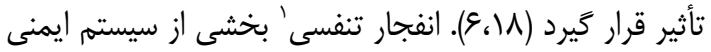

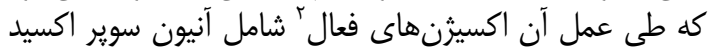

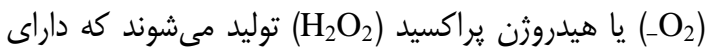

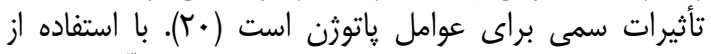

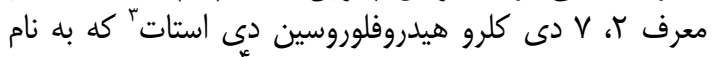

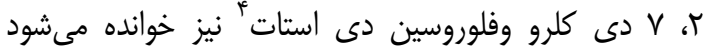

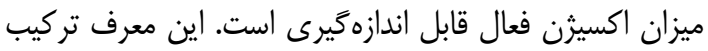

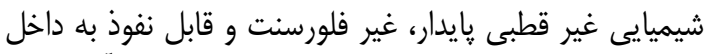

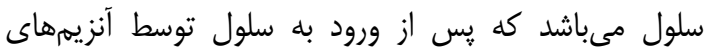

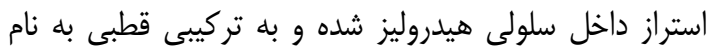

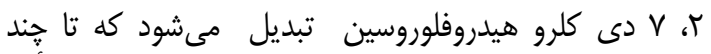

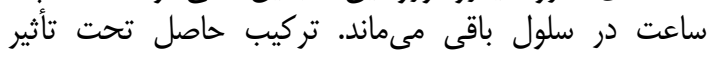

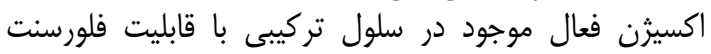

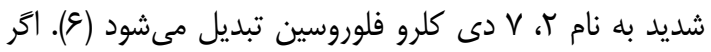

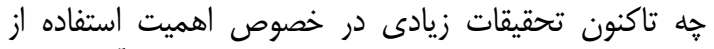

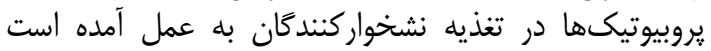

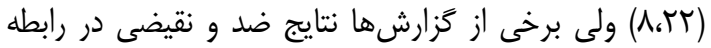

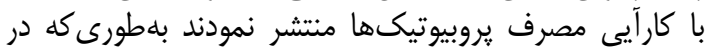

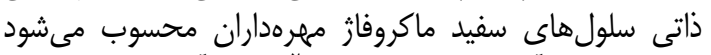

مقدمه

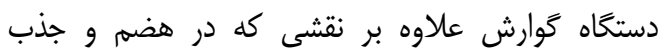

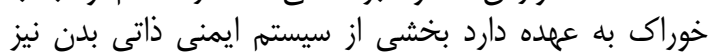

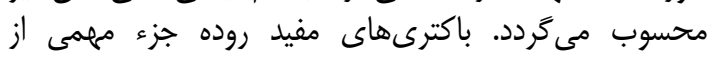

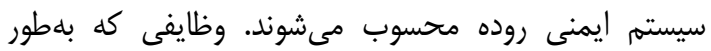

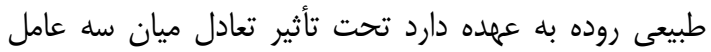

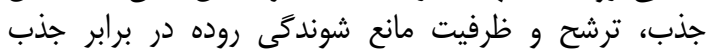

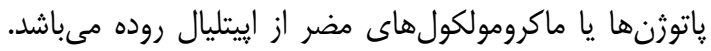

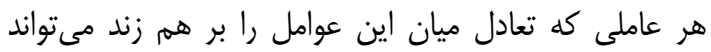

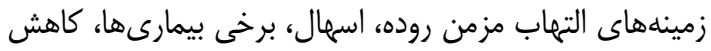

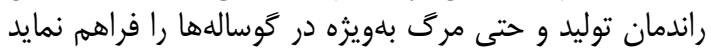

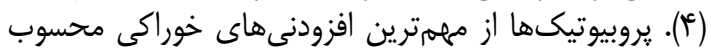

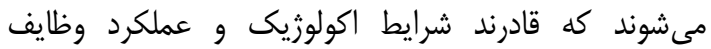

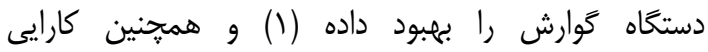

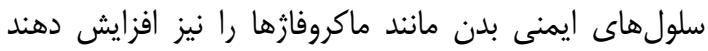

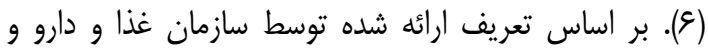

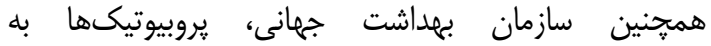

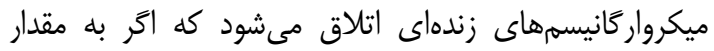

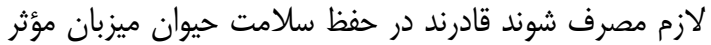

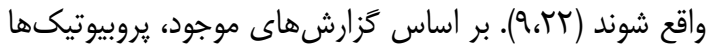

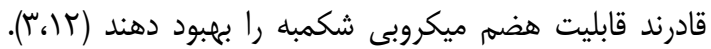

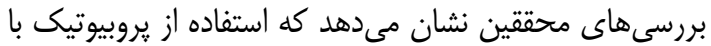

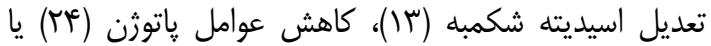

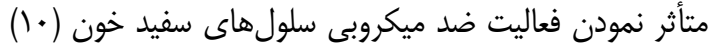

1- Oxygen brust

3- Dichlorodihydrofluorescein diacetate (DCFH-DA)

2- Reactive oxygen species

4- Dichloro fluorescin diacetate 
انفجار تنفسى براى فعاليت ضد ياتوزنى سلولهاى سفيد خون كوسالههاى نزاد كردى ايران مىباشد.

\section{مواد و روشها}

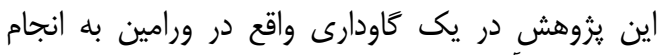

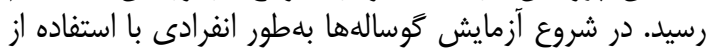

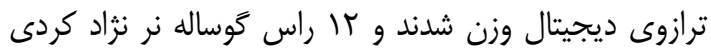

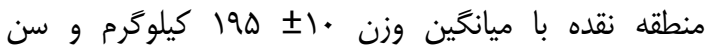

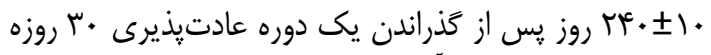

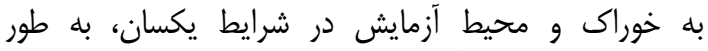

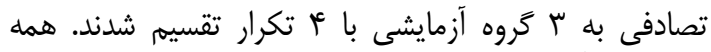

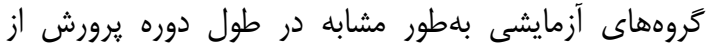

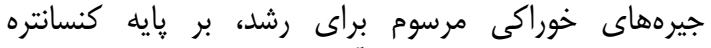

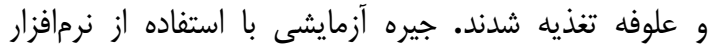

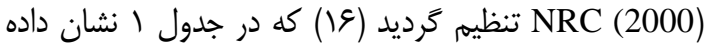

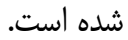

برخى از گزارشها مشاهده مى گردد كه ميزان عملكرد دام

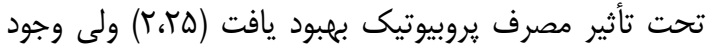

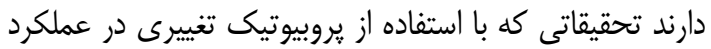

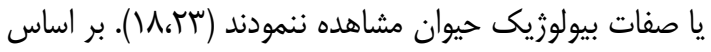

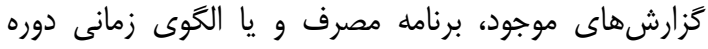

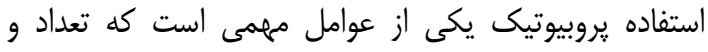

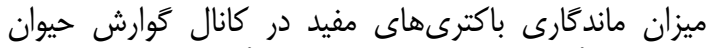

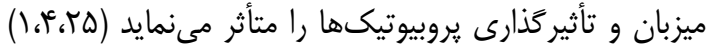

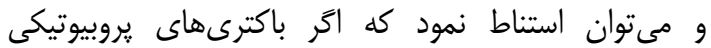

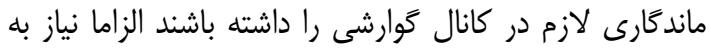

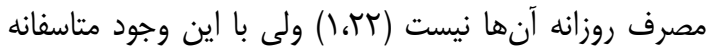

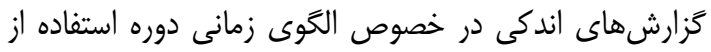

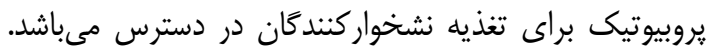

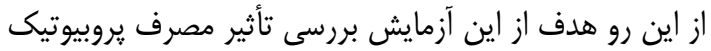

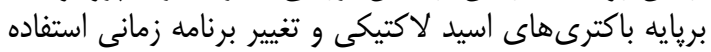

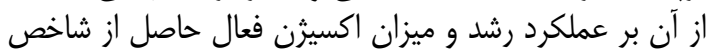

Table 1. Ingredients and chemical composition of control diet

جدول ا- اجزاء مواد خوراكى و تركيب شيميايى جيره كنترل

\begin{tabular}{|c|c|}
\hline (درصد) & مواد خوراكى \\
\hline 14 & دانه جو \\
\hline 1 . & دانه ذرت \\
\hline$r / 4$ & كنجاله سويا \\
\hline 1 . & سبوس كندم \\
\hline . $/ 94$ & مكمل معدنى و ويتامين \\
\hline ./VY & كربنات كلسيم \\
\hline.$/ 4$ & نمى \\
\hline tr & كاه كندم \\
\hline זr & يونجه \\
\hline & تركيب شيميايى بر حسب ماده خشى \\
\hline$\Lambda 9 / 4$ & ماده خشك (درصد) \\
\hline זו & يروتئين خام (درصد) \\
\hline $41 / 9$ & الياف نامحلول در شوينده خنثى (درصد) \\
\hline$\cdot / \mathrm{AV}$ & كلسيم (درصد) - مل \\
\hline$T / 1$ & انرزى قابل متابوليسم (مكاكالرى در هر كيلوكَرم) \\
\hline
\end{tabular}

اسيد لاكتيك و بلدون يوشش باكتريايى مىباشد. اين

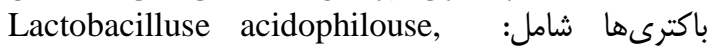
Lactobacilluse casei, Bifidobacterium thrmophilum, Enterococcuse faecium קند سال اخير نيز تأثير آنها بلهنوان يروبيوتيك در كانال

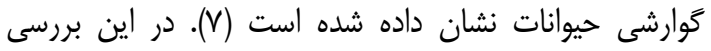

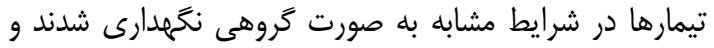

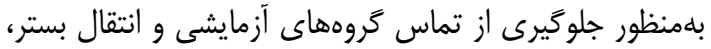

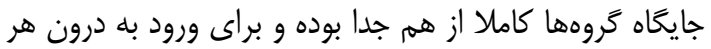

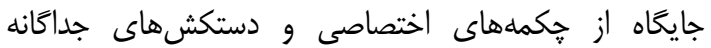

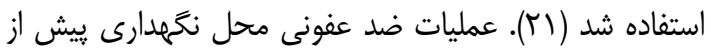

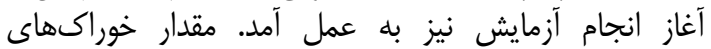

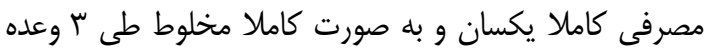

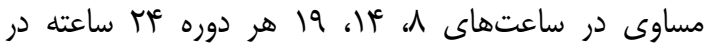

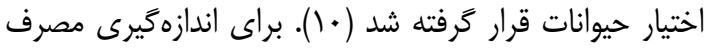

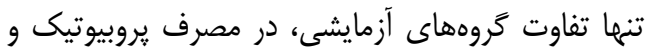

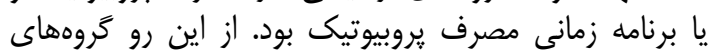

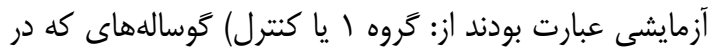

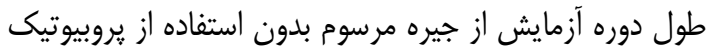

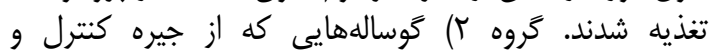

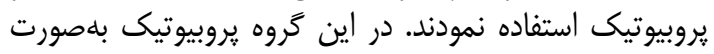

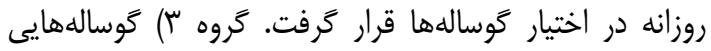

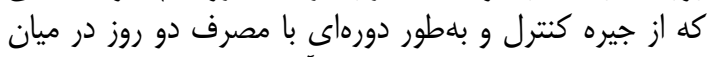

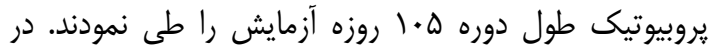

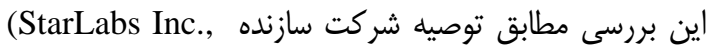
سه كَرم يروبيوتيك با نام تجارى (Plarksdale, MO, USA)

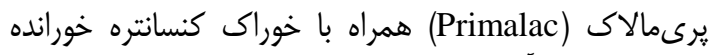

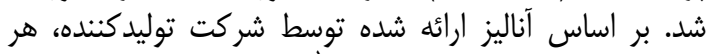

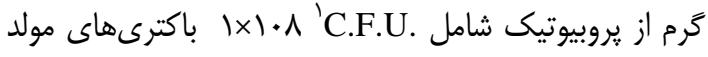




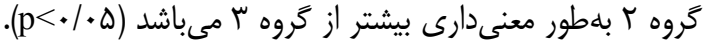

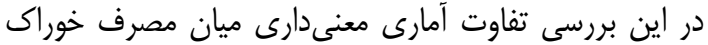

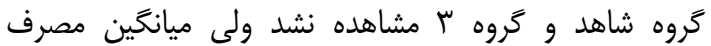

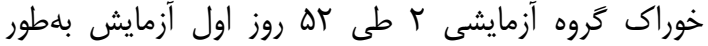

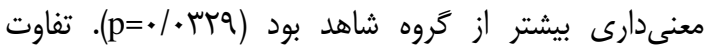

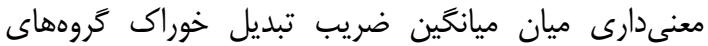

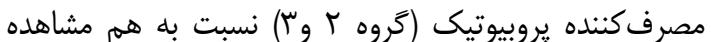

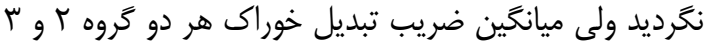

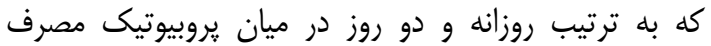

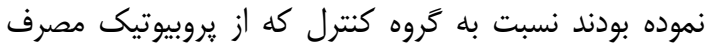

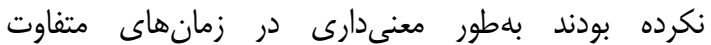

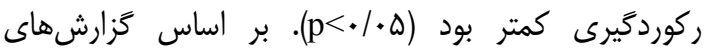

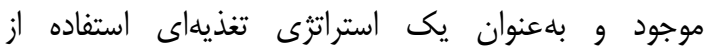

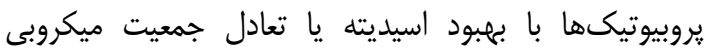

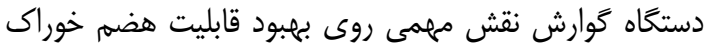
(IIIV)

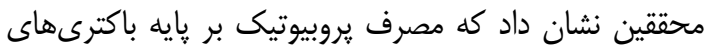

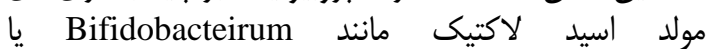
Enterococcuse مىباشند بواسطه ترشح اسيد لاكتيك در تركئ تعديل اسيدايته محيط دستخاه كوارش تأثير داشته و در نتيجه آن آن اكوسيسته

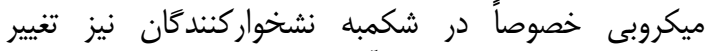

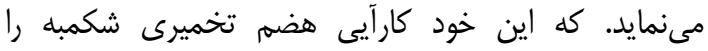

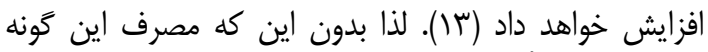

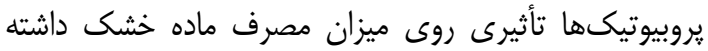

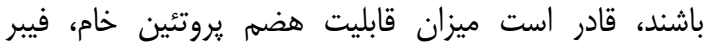

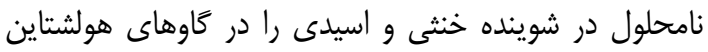

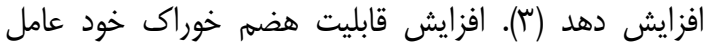

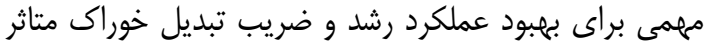

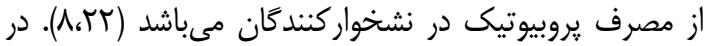

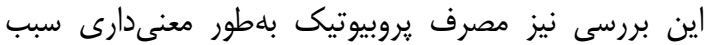

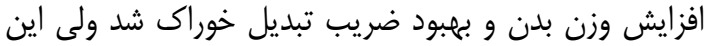

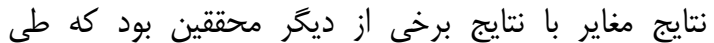

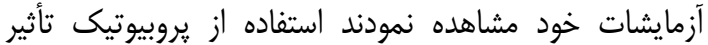

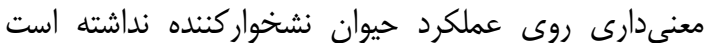

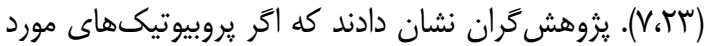

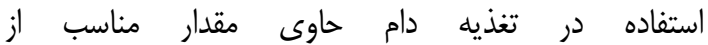

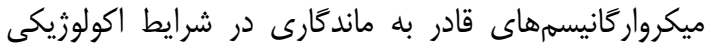

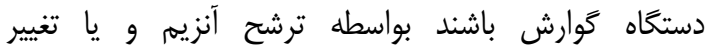

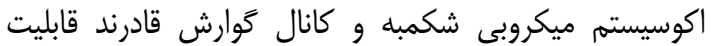

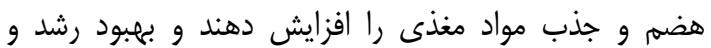

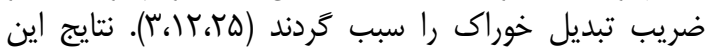

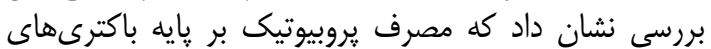

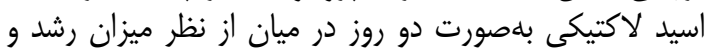

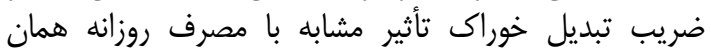

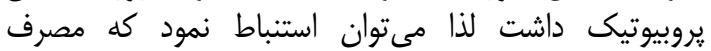
يروبيوتيكى مورد آزمون ماندكارى و و تأثيركذارى لازيك لازم درد

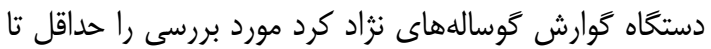

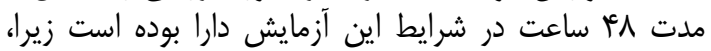

خوراك روزانه مقدار خوراك باقى مانده از روز قبل در نايلون

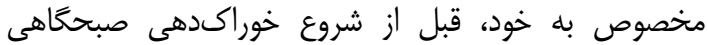

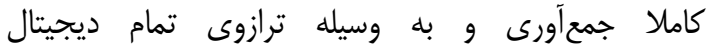

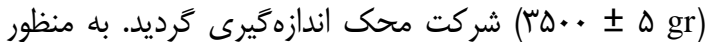

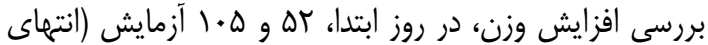

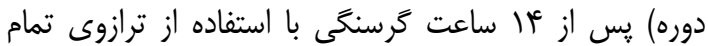

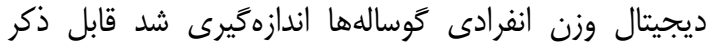

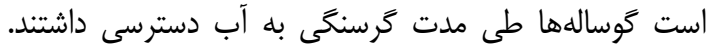

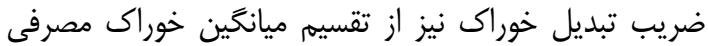

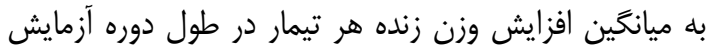

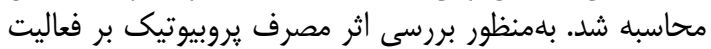

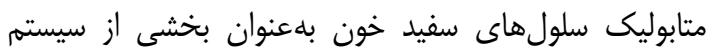

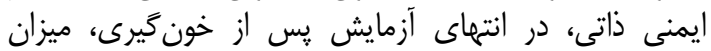

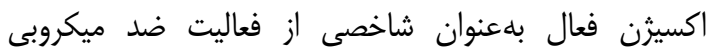

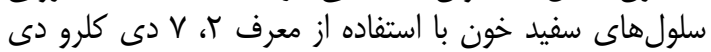

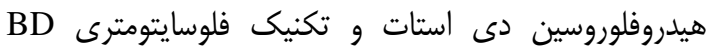
FACSCaliburtm Flow) Cytometer- BD Biosciences Co

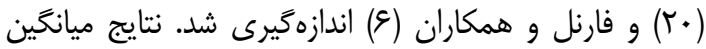

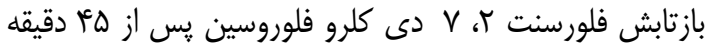

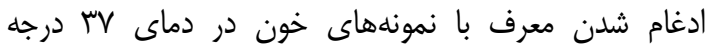

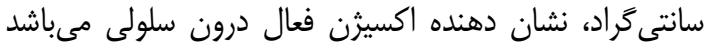

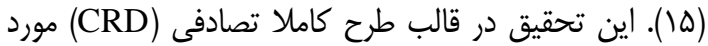
تجزيه و تحليل أمارى قرار داده شد. مدل آمارى به صاله صورت

$\mathrm{Yij}=\mu+\sigma \mathrm{j}+\varepsilon \mathrm{ij}$

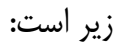

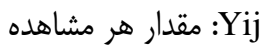

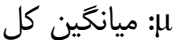

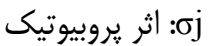

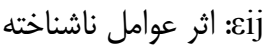

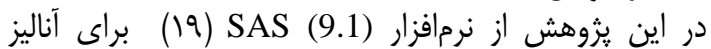

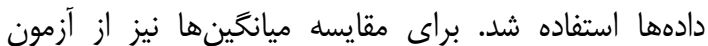
دانكن در سطح احتمال هـ/• استفاده شد.

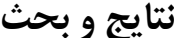

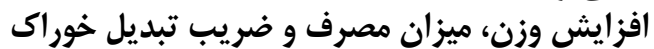

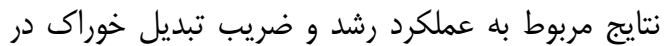

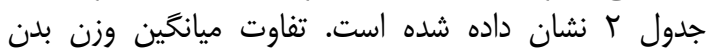

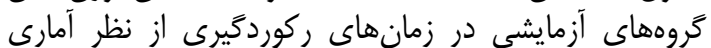

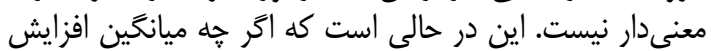

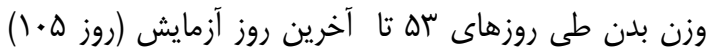

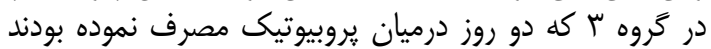

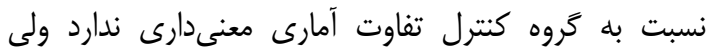

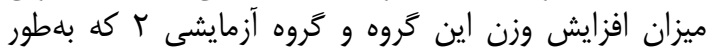

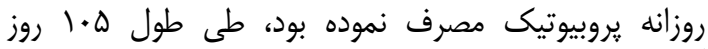

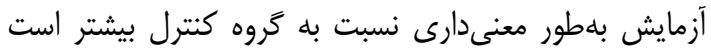

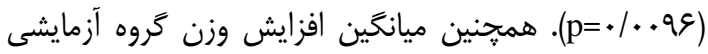

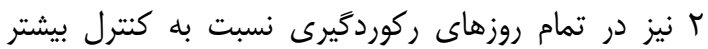

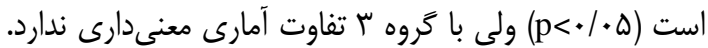
در همه دورههاى ركوردكيرى ميانكين مصرف خوات خوراك در 


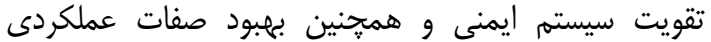

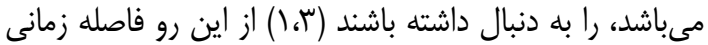

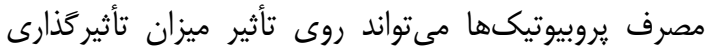

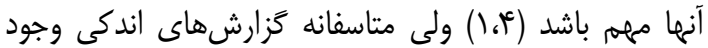

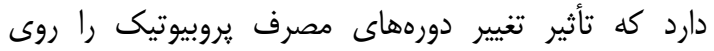

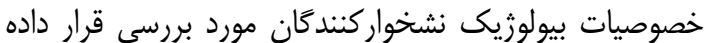

باشد.
نشان داده شده است كه ميكرواركانيسمهايى كه بهاعنوان

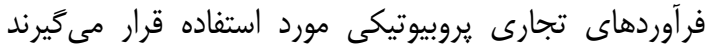

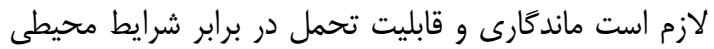

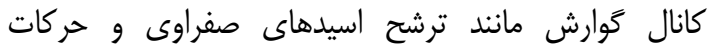

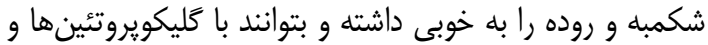

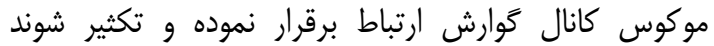

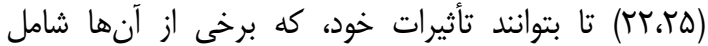
تحريك در افزايش جمعيت باكترىهاى مفيد كانال كوارشى،

جدول r- عملكرد رشد، مصرف و ضريب تبديل خوراك در گوسالههاى آزمايشى Table 2. Growth performance, feed intake and feed convertion rate of experimental calves

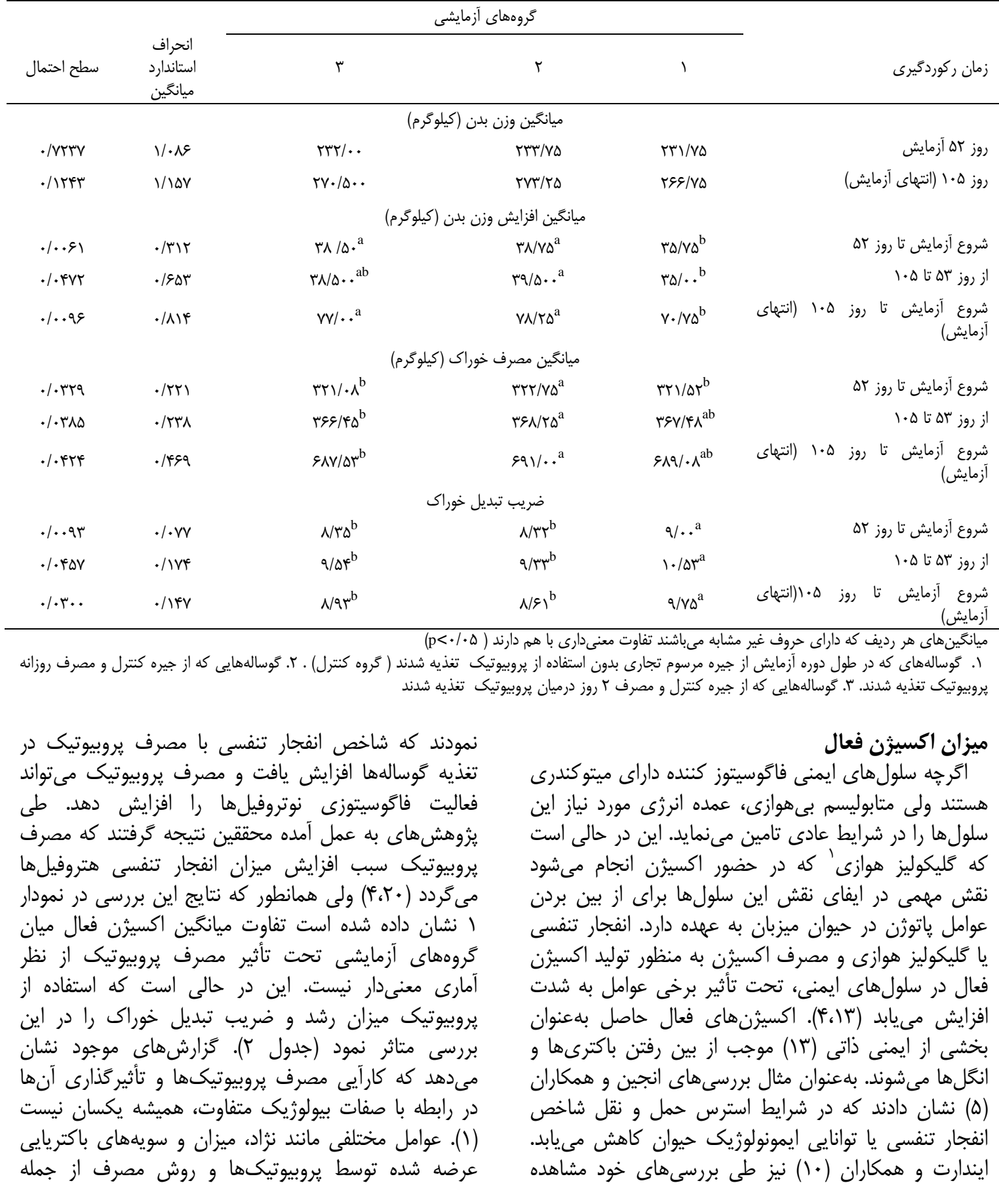


مىباشيد و مىتوان به جاى مصرف روزانه، دو روز در ميان از

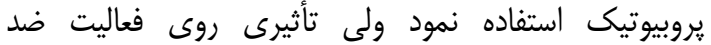
ميكروبى سلولهاى سفيد خون كوسالههاى نزاد كرد ندارد.
مهمترين عوامل مؤثر بر تأثيركذارى يروبيوتيكها شناحته

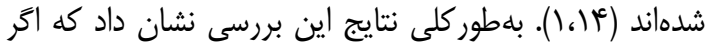

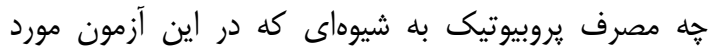

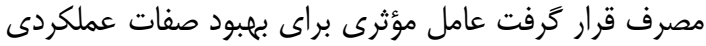

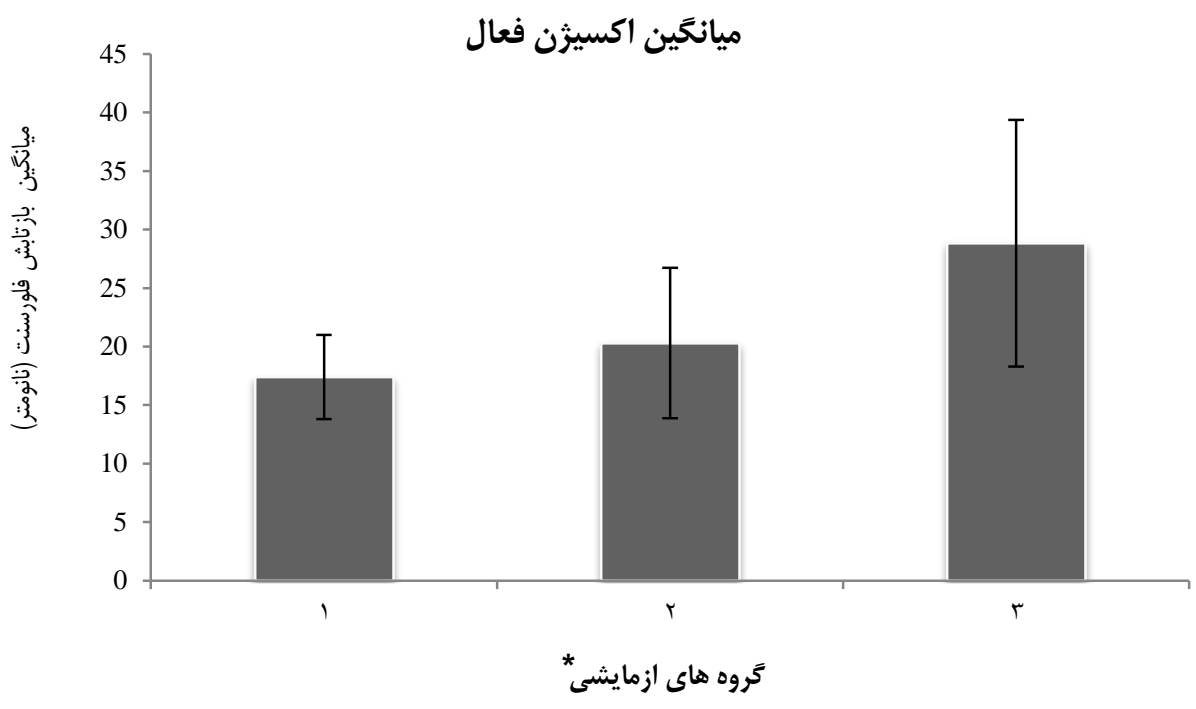

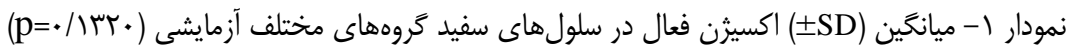

Figure 1. Reactive oxygen (mean \pm SD) in white blood cell in different experimental group (p=0.1320)

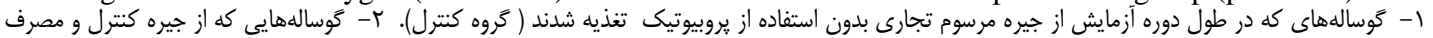

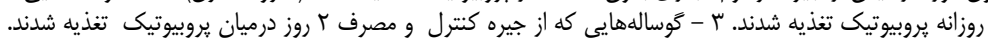

1. Aliakbarpour, H.R., M. Chamani, G. Rahimi, A.A. Sadeghi and D. Qujeq. 2013. The Bacillus subtilis and Lactic Acid Bacteria Probiotics Influences Intestinal Mucin Gene Expression, Histomorphology and Growth Performance in Broilers. Asian-Australian Journal of Animal Science, 25: 1285-1293.

2. Azimzadeh, V., A. Assadi-Alamouti, A. Khadem, M. Bagheri Varzaneh and J. Mohammad Moradi. 2015. Effects of Supplementation of a Symbiotic Product on Growth Performance and Health of Holstein Calves. Research on Animal Production, 6: 105-114 (In Persion).

3. Boyd, J., J. West and J. Bernard. 2011. Effects of the addition of direct-fed microbials and glycerol to the diet of lactating dairy cows on milk yield and apparent efficiency of yield. Journal of Dairy Science, 94: 4616-4622.

4. Corcionivoschi, N., D. Drinceanu, I.M. Pop, D. Stack, L. Lavinia Stef, C. Julean and B. Bourke. 2010. The Effect of Probiotics on Animal Health. Animal Science and Biotechnology, 43: 35-41.

5. Engen, N.K.V., M.L. Stock, T. Engelken, R.C. Vann, L.W. Wulf, L.A. Karriker, W.D. Busby, J. Lakritz, A.J. Carpenter, B.J. Bradford, W.H. Hsu, C. Wang and J.F. Coetzee. 2014. Impact of oral meloxicam on circulating physiological biomarkers of stress and inflammation in beef steers after long-distance transportation. Journal of Animal Science, 92: 498-510.

6. Farnell, M.B., A.M. Donoghue, F.S. Santos, P.J. Blore, B.M. Hargis, G. Tellez and D.J. Donoghue. 2006. Upregulation of Oxidative Burst and Degranulation in Chicken Heterophils Stimulated with Probiotic Bacteria. Poultry Science, 85: 1900-1906.

7. Francia, A.D., F. Masucci, M.L. Varricchio, A. Bilancione and V. Proto. 2007. Effect of Enterococcus faecium SF68 on growth performance and in vivo digestibility in buffalo calves. Italian Journal of Animal Science, 6: 299-301.

8. Frizzoa, L.S., M.V. Zbruna, L.P. Sotoa and M.L. Signorinib. 2011. Effects of probiotics on growth performance in young calves: A meta-analysis of randomized controlled trials. Animal Feed Science and Technology, 169: 147-156.

9. Hosseinabadi, M., M. Dehghan-Banadaky and A. Zali. 2013. The Effect of feeding of bacterial probiotic in milk or starter on growth performance, health, blood and rumen parameters of suckling calves. Research on Animal Production, 4: 57- 69 (In Persion).

10. Indart, M., S. Cerone, E.N. Esteban, G.D. Yaniz, A.G. Inza, H. Landi, S. Mogni and L. Igarza. 2012. Multispecies Multistrain Probiotic Effects on Calves Development and Health. Open Journal of Veterinary Medicine, 2: 225-229. 
4.

اثر زمانبندى مصرف يروبيوتيك بر پايه باكترىهاى اسيد لاكتيكى بر عملكرد و

11. Kritas, S.K., T. Marubashi, G. Filioussis, E. Petridou, G. Christodoulopoulos, A.R. Burriel, A. Tzivara, A. Theodoridis and M. Pískoriková. 2015. Reproductive performance of sows was improved by administration of a sporing bacillary probiotic (Bacillus subtilis C-3102). Journal of Animal Science, 93: 405-413.

12. Le, O., P. Dart, K. Harper, D. Zhang, B. Schofield, M. Callaghan, A. Lisle, A. Klieve and D. McNeill. 2016. Effect of probiotic Bacillus amyloliquefaciens strain $\mathrm{H} 57$ on productivity and the incidence of diarrhoea in dairy calves. Animal Production Science, 57: 912-919.

13. Lettat, A., P. Nozière, M. Silberberg, D.P. Morgavi, C. Berger and C. Martin. 2012. Rumen microbial and fermentation characteristics are affected differently by bacterial probiotic supplementation during induced lactic and subacute acidosis in sheep. BMC Microbiology, 12: 142-148.

14. Mountzouris, K.C., P. Tsitrsikos, I. Palamidi, A. Arvaniti, M. Mohnl. G. Schatzmayr and K. Fegeros. 2010. Effect of probiotic inclusion levels in broiler nutrition on growth performance, nutrient digestibility, plasma immunoglobulins, and cecal microflora composition. Poultry Science, 89: 58-67.

15. Nouri, S., M. Movahedin and Z. Mazaheri. 2014. Effects of mouse paternal age on sperm parameters, reactive oxygen species levels, intraspermic antioxidants and in vitro fertilization results. Modares Journal of Medical Sciences, 17: 93-103 (In Persion).

16. NRC. 2000. Nutrient requirements of beef cattle, $7^{\text {th }}$ revised. National Academy Press, Washington, DC.

17. Rezaee, M.T., M.T. Rezaeian, M.T. Moradi Shahrebabak and S.A.N. Mirhadi. 2006. The Effects of strain and doses of saccharomyces cerevisiae supplementation on performance, total rumen bacterial population and blood serum metabolites in male holstein calves. Journal of Veterinary Research, 61: 63-69 (In Persion).

18. Rezaie-Khormenany, B., S. Karimi Dehkordi and A. Mohebi. 2016. Effect of different sources of probiotics on performance and some blood parameters of indigenous kid's. Iranian Journal of Animal Science, 47: 313-321 (In Persion).

19. SAS Institute. 2003. SAS User's Guide.Version 9.1 ed. SAS Institute Inc., Cary, NC, USA.

20. Stringfellow, K., D. Caldwell, J. Lee, M. Mohnl, R. Beltran, G. Schatzmayr, S. Fitz-Coy, C. Broussard and M. Farnell. 2011. Evaluation of probiotic administration on the immune response of coccidiosis-vaccinated broilers. Poultry Science, 90: 1652-1658.

21. Sun, P. and J.Q. Wang. 2010. Effecs of Bacillus subtilis natto on performance and immune function of preweaning calves. American Dairy Science Assoclation, 93: 5851-5855.

22. Uyeno, Y., S. Shigemori and T. Shimosato. 2015. Effect of probiotics/prebiotics on cattle health and productivity. Microbes and Environments, 30: 126-132.

23. Whitley, N.C., D. Cazac, B.J. Rude, D.J. Brien and S. Parveen. 2009. Use of a commercial probiotic supplement in meat goats. Journal of Animal Science, 87: 723-728.

24. Wisener, L., J. Sargeant, A. O'Connor, M. Faires and S. Glass-Kaastra. 2014. The use of direct-fed microbials to reduce shedding of Escherichia coli O157 in beef cattle: a systematic review and metaanalysis. Zoonoses and Public Health, 62: 7589.

25. Yirga, H. 2015. The use of probiotics in animal nutrition. Journal of Probiotic and Health. ISSN: 2329-8901 JPH, an open access journal, 3: 1-10. 


\title{
The Effect of Lactic Acid Bacteria Based Probiotic use Schedule on Growth Performance and Blood Cell Antimicrobial Activity in Kurd Calves
}

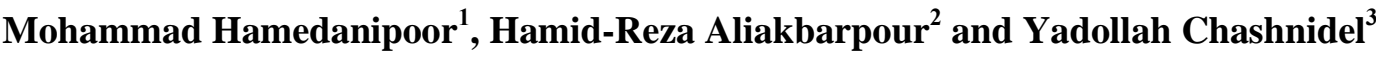

\footnotetext{
1- Gradute, Department of Veterinary Medicin, Babol Branch, Islamic Azad University, Babol, Iran 2- Department of Veterinary Medicin, Babol Branch, Islamic Azad University Babol, Iran (Corresponding auther: aliakbarpour@hotmail.com)

3- Associate Professor, Department of Animal Science, Sari Agricultural Sciences and Natural Resources University Received: March 6, 2018 Accepted: June 24, 2018
}

\begin{abstract}
The aim of this study was to examine the effect of Lactic acid bacteria based probiotic and its use schedule on Kurd male calves' growth performance and white blood cell antimicrobial activity. The experimental period was 105 days after calves adapted to new environmental conditions. This study was carried out by 12 calves with an average body weight of $195 \pm 10 \mathrm{~kg}$ and age of $240 \pm 10$ days in a completely randomized design with three treatments and three replicate. Experimental groups included: 1) the control group was fed on a commercial basal diet (without probiotic). 2) was fed on basal diet and daily usage probiotic 3) was fed on basal diet and probiotic usage a day then skip 2 days and repeated. Growth performance and feed conversion rate were determined by measuring calves weight and feed intake on day 0,52 and 105 of experiment after $14 \mathrm{~h}$ feed deprivation. For measurement of reactive oxygen species as anitimicrobial activity index in white Blood Cells, blood samples were taken from all of the calves at the end of experiment. Although body weight gain and feed conversion rate was not significantly different among any of the treatments 2 and 3 but increased and decreased respectively compared to control group in end of experiment $(\mathrm{p}<0.05)$. Feed intake significantly increased in treatment 3 compared $2(\mathrm{p}=0.0424)$. No differences in reactive oxygen mean were observed in calves fed probiotic-supplemented diets and control group. According to this results dietary supplementation with probiotic did not affect white blood cell antimicrobial activity although improved Kurd calves growth performance and feed conversion rate, also daily usage of probiotic can be replaced by usage a day then skip 2 days and repeated schedule.
\end{abstract}

Keywords: Calf, Growth, Probiotic, Reactive oxygen 